\section{Investigating chemoresistance to improve sensitivity of childhood T-cell acute lymphoblastic leukemia to parthenolide}

\author{
Benjamin C. Ede, ${ }^{1}$ Rafal R Asmaro, ${ }^{1}$ John P. Moppett, ${ }^{1,2}$ Paraskevi Diamanti ${ }^{1,3}$ \\ and Allison Blair ${ }^{1,3}$ \\ ${ }^{1}$ School of Cellular and Molecular Medicine, University of Bristol; ${ }^{2}$ Bristol Royal Hospital for \\ Children and ${ }^{3}$ Bristol Institute for Transfusion Sciences, NHS Blood and Transplant, UK
}

\section{ABSTRACT}

$\longrightarrow$ urrent therapies for childhood T-cell acute lymphoblastic leukemia have increased survival rates to above $85 \%$ in developed countries. Unfortunately, some patients fail to respond to therapy and many suffer from serious side effects, highlighting the need to investigate other agents to treat this disease. Parthenolide, a nuclear factor kappa $(\kappa) B$ inhibitor and reactive oxygen species inducer, has been shown to have excellent anti-cancer activity in pediatric leukemia xenografts, with minimal effects on normal hemopoietic cells. However, some leukemia initiating cell populations remain resistant to parthenolide. This study examined mechanisms for this resistance, including protective effects conferred by bone marrow stromal components. T-cell acute leukemia cells co-cultured with mesenchymal stem cells demonstrated significantly enhanced survival against parthenolide $(73 \pm 11 \%)$ compared to cells treated without mesenchymal stem cell support $(11 \pm 9 \%)$. Direct cell contact between mesenchymal cells and leukemia cells was not required to afford protection from parthenolide. Mesenchymal stem cells released thiols and protected leukemia cells from reactive oxygen species stress, which is associated with parthenolide cytotoxicity. Blocking cystine uptake by mesenchymal stem cells, using a small molecule inhibitor, prevented thiol release and significantly reduced leukemia cell resistance to parthenolide. These data indicate it may be possible to achieve greater toxicity to childhood T-cell acute lymphoblastic leukemia by combining parthenolide with inhibitors of cystine uptake.

\section{Introduction}

The introduction of contemporary therapies for childhood T-cell acute lymphoblastic leukemia (T-ALL) has resulted in remission rates that are closer to that of B-cell precursor (BCP) ALL but survival rates remain lower and 15-20\% of children with T-ALL die from relapsed/refractory disease. ${ }^{1}$ Patients with high-risk disease or those who relapse often receive more intensive treatment, making them more susceptible to toxicity and long-term secondary complications. ${ }^{2}$ This highlights the need to investigate other agents to treat this disease.

It has been demonstrated that numerous cancers generate high levels of reactive oxygen species (ROS) compared to healthy tissue counterparts, where ROS levels are normally maintained in a tightly controlled manner. ${ }^{3}$ In T-ALL, ROS levels have been shown to be heightened, and this can inactivate phosphatase and the tensin homolog (PTEN), promoting leukemia cell survival. ${ }^{4}$ In human T-ALL, ROS levels are restrained by downregulation of protein kinase $\mathrm{c}$ theta (PKC $\theta$ ) caused by NOTCH-1, a commonly activated mutation in T-ALL. ${ }^{5}$ However, if ROS stress levels are pushed above a certain threshold, cell death is forced to occur. ${ }^{3}$ Therefore, ROS promoting drugs may be an effective way of targeting cancer cells. Parthenolide (PTL) has been previously shown by ourselves and others to be a promising therapeutic agent for blood cancers. ${ }^{6.8}$ Importantly, it has limited effects
Haematologica 2018

Volume 103(9):1493-1501

\section{Correspondence:}

allison.blair@bristol.ac.uk

Received: December 18, 2017.

Accepted: May 10, 2018.

Pre-published: May 17, 2018.

doi:10.3324/haematol.2017.186700

Check the online version for the most updated information on this article, online supplements, and information on authorship \& disclosures: www.haematologica.org/content/103/9/1493

(C)2018 Ferrata Storti Foundation

Material published in Haematologica is covered by copyright. All rights are reserved to the Ferrata Storti Foundation. Use of published material is allowed under the following terms and conditions:

https://creativecommons.org/licenses/by-nc/4.0/legalcode. Copies of published material are allowed for personal or internal use. Sharing published material for non-commercial purposes is subject to the following conditions:

https://creativecommons. org//icenses/by-nc/4.0/legalcode, sect. 3. Reproducing and sharing published material for commercial purposes is not allowed without permission in writing from the publisher. 
Table 1. Patients' sample characteristics.

\begin{tabular}{|c|c|c|c|c|c|}
\hline Patient N. & Karyotype & $\operatorname{Sex}$ & Age (vears) & Disease status at biopsy & MRD risk status ${ }^{a}$ \\
\hline 1 & $46 X Y$ & M & 7 & Diagnosis & Risk \\
\hline 2 & $\mathrm{t}(1 ; 14)$ & M & 3 & Diagnosis & Risk \\
\hline 3 & $46 X Y$ & M & 17 & Diagnosis & High \\
\hline 4 & $\mathrm{t}(8 ; 14)$ & M & 3 & Diagnosis & Low \\
\hline 5 & $\mathrm{t}(11 ; 14), \operatorname{add}(17)$ & M & 2 & Relapse & High \\
\hline 6 & Runxl rearr & M & 3 & Diagnosis & N/A \\
\hline 7 & $46 X Y$ & M & 6 & Diagnosis & Low \\
\hline 8 & add (4), add (9) & M & 14 & Diagnosis & High \\
\hline 9 & del (4), del (9) & M & 9 & Diagnosis & Low \\
\hline 10 & add (7), add (9), add (14) & M & 4 & Diagnosis & Low \\
\hline
\end{tabular}

All patients treated on UKALL2011 protocol, except patients 3,5 and 8 who were treated on UKALL 2003. ${ }^{a}$ Minimal residual disease (MRD) risk status at Day 29, on respective treatment protocols. N: number; M: male.

on normal cells at the doses required to kill cancer cells. PTL can target cancer cells via numerous mechanisms, such as inhibition of nuclear factor (к)B, p53 activation and ROS stress. ${ }^{67}$ However, the mechanism of PTL toxicity to T-ALL has not been defined.

Parthenolide has been shown to be very effective against childhood T-ALL in vivo, with elimination of the disease and restoration of murine hemopoiesis in

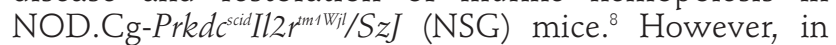
mice engrafted with different leukemia initiating cell populations from 2 of 9 T-ALL cases, disease progression was delayed rather than eliminated, indicating variable sensitivity of certain subpopulations to PTL. Reasons for the differences in sensitivity may be due to the effect of the in vivo microenvironment. Bone marrow (BM) stromal cells release cysteine for uptake by chronic lymphocytic leukemia (CLL) cells, driving anti-oxidative glutathione synthesis, which provides protection against ROS generating chemotherapeutic agents, such as fludarabine and oxiplatin. ${ }^{9}$

Mesenchymal stem cells (MSC) are key constituents of the BM microenvironment and have been shown to enhance protection against certain drugs in T-ALL cell lines $^{10}$ and primary samples from patients with B-ALL, acute myeloid leukemia (AML) and CLL.,11-13 Co-culture of T-ALL cell lines with MSC enhanced resistance to the anthracycline idarubicin. ${ }^{10}$ However, the role of ROS in stromal cell mediated protection in childhood ALL has not been reported. As we had previously reported resistance to PTL in T-ALL cases, in this study the cytotoxic and ROS inducing effects of the drug on primary T-ALL cells in the presence of MSC were examined to increase our understanding of PTL resistance.

\section{Methods}

\section{T-ALL and normal samples}

Bone marrow samples from 10 children, aged 2-17 years (median 5 years), diagnosed with T-ALL at presentation or relapse were collected with informed consent and approval of University Hospitals Bristol NHS Trust and London Brent Research Ethics Committee (Table 1). Mononuclear cells (MNC) were separated via density gradient centrifugation using FicollHypaque (Sigma-Aldrich, Gillingham, UK). MNC were sus- pended in $90 \%$ fetal calf serum (FCS, Thermo Scientific, Paisley, UK) and 10\% dimethyl sulfoxide (DMSO, Origen Biomedical, Solihull, UK) and stored in liquid nitrogen prior to use. Samples from patients with a range of karyotypic abnormalities, diagnostic age and minimal residual disease (MRD) status were investigated.

Bone marrow from a consenting healthy donor was used as a source of MSC. See the Online Supplementary Appendix for full details of MSC expansion and characterization.

\section{Cytotoxicity assays}

T-cell acute lymphoblastic leukemia cells were plated in duplicate (for each drug concentration tested) at $1.2 \times 10^{5} \mathrm{cells} / \mathrm{mL}$ in RPMI 1640 medium (Sigma-Aldrich) containing 20\% FCS, 1\% Lglut and $1 \%$ Pen/Strep, hereafter referred to as suspension medium. Drugs used for assays were: PTL (Enzo Life Sciences, Exeter, UK) at 1-10 $\mu \mathrm{M}, \mathrm{N}$-acetyl cysteine (NAC, Sigma-Aldrich) at $15 \mathrm{mM}$ and $30 \mu \mathrm{M}$, and sulfasalazine (SSZ, Sigma-Aldrich) at $300 \mu \mathrm{M}$, all prepared in suspension medium. For co-culture experiments, $5 \times 10^{4} \mathrm{MSC} / \mathrm{mL}$ were seeded per well and left to adhere for 24 hours (h). MSC medium was removed and replaced with suspension media containing $1 \times 10^{5}$ T-ALL cells/mL. T-ALL cells were left to settle for $1 \mathrm{~h}$ and then treated with $10 \mu \mathrm{M}$ PTL with or without $300 \mu \mathrm{M}$ SSZ, for $24 \mathrm{~h}$. After treatment, non-adherent cells were removed and stained with annexin-V conjugated to fluorescein isothiocyante (Miltenyi Biotec) for 10 minutes (min). Cells were washed and stained with propidium iodide (PI, Miltenyi Biotec) prior to flow cytometric analysis. See Online Supplementary Figure S1 for details.

For transwell separation experiments, $1 \times 10^{5} \mathrm{MSC} / \mathrm{mL}$ were seeded per well in MSC media and left to adhere for $24 \mathrm{~h}$. MSC medium was removed and replaced with suspension medium. T-ALL cells were seeded at $2 \times 10^{5}$ cells $/ \mathrm{mL}$ onto Costar Transwell inserts $(0.4 \mu \mathrm{M}$ pore size, Corning Life Sciences, Ewloe, UK) above the adherent MSC. T-ALL cells were left to settle for $1 \mathrm{~h}$ and then treated with PTL with or without SSZ for $24 \mathrm{~h}$. After treatment, cells in transwell inserts were removed and viability was assessed by flow cytometry, as above.

\section{Reactive oxygen species detection}

Cells were treated with $5 \mu \mathrm{M}$ of the redox sensitive probe 5 (and-6)-chloromethyl-2',7'-dichlorodihydrofluorescein diacetate (CM-H2DCFDA, Thermo Fisher Scientific) for $30 \mathrm{~min}$ at $37^{\circ} \mathrm{C}$. Cells were treated with PTL, then immediately analyzed 
A

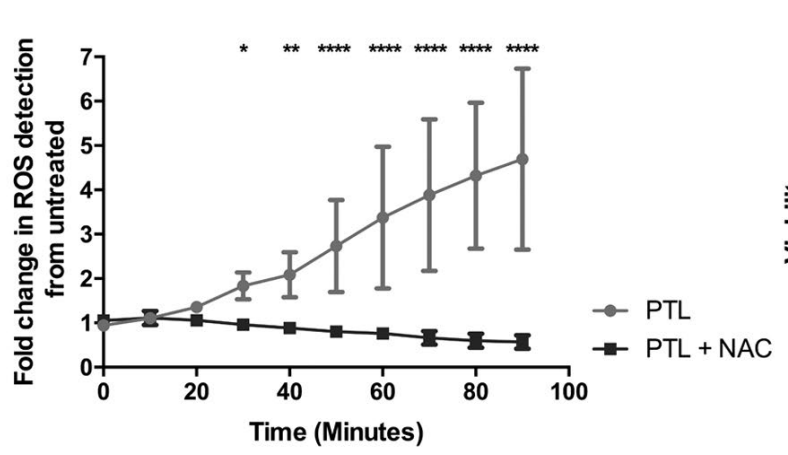

B

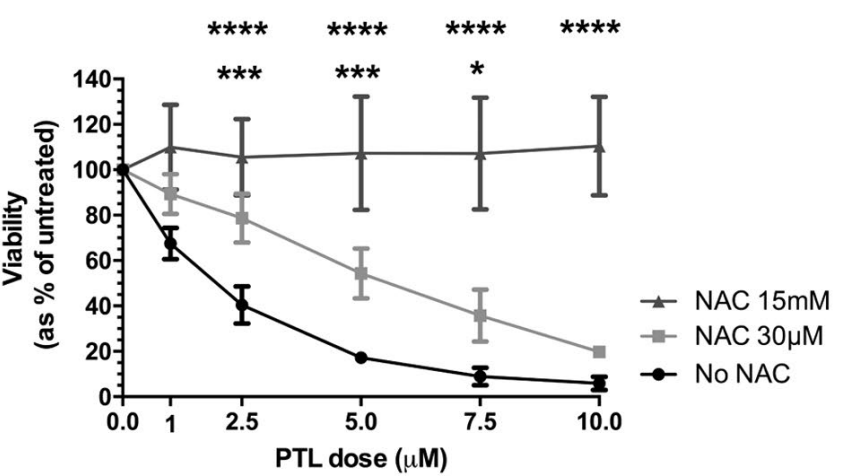

C

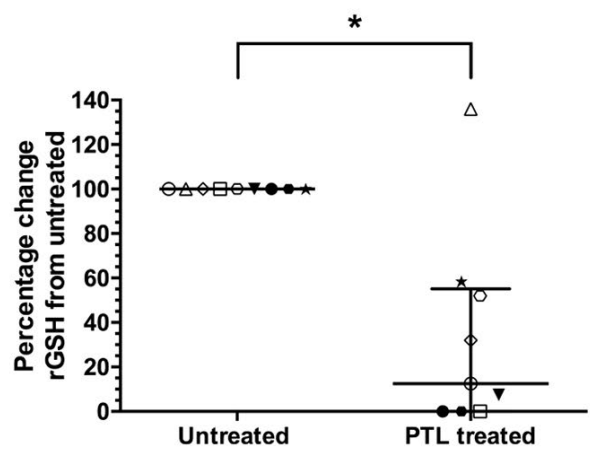

Figure 1. Parthenolide (PTL) induces changes in reactive oxygen species (ROS) and reduced glutathione ( $\mathrm{rGSH}$ ) levels can be reversed by $\mathrm{N}$-acetyl cysteine (NAC). (A) T-cell acute lymphoblastic leukemia (T-ALL) samples (patients 1, 3, 5, and 9) were pre-incubated with the ROS sensitive probe CMH2DCFDA and ROS accumulation was measured in live cells over 90 minutes by flow cytometry in response to $10 \mu \mathrm{M}$ PTL or with PTL+15 mM NAC. Fold change in median fluorescence intensity (MFI) compared to untreated cells is shown; data represent mean \pm Standard Deviation (SD). Asterisks represent significant differences between PTL and PTL+15 mM NAC. (B) Dose response curve of patient samples 2, 5, 8, and 10 that had been treated with PTL only (1-10 $\mu \mathrm{M}), \mathrm{PTL}+30 \mu \mathrm{M}$ NAC, or PTL+15mM NAC; data represent mean \pm SD. Top row of asterisks represent significant differences between PTL alone versus PTL+15mM NAC; bottom row of asterisks represent significant differences between PTL alone versus PTL+30 $\mu \mathrm{M}$ NAC. (C) GSH levels were measured in T-ALL samples (patients 1-9) after treatment with PTL $(10 \mu \mathrm{M})$ for 1 hour and compared with those in untreated cells. Symbols represent the mean \% change from untreated cells in replicate samples. Each symbol represents an individual patient (see Online Supplementary Figure S1 for patient symbol key). Lines represent median and interquartile range. Results were analyzed by two-way ANOVA (A and $B$ ) or paired $t$-test $(C) .{ }^{*} P \leq 0.05, * * P \leq 0.01, * * * P \leq 0.001, * * * * P \leq 0.0001$. over a 90 min period by staining with PI and measuring the median fluorescence intensity (MFI) of the ROS probe by flow cytometry.

\section{Reduced glutathione detection}

The level of reduced glutathione (rGSH) was detected using the GSH/GSSG-Glo luminescence assay using a plate reader (GloMax, both Promega, Chilworth, UK). Levels of rGSH were quantified in $2 \times 10^{4}$ live T-ALL cells either untreated or treated with $10 \mu \mathrm{M}$ PTL for $1 \mathrm{~h}$.

\section{Thiol detection}

The thiol concentration in media was detected using a 5,5'dithiobis-(2-nitrobenzoic) (DTNB) assay. MSC were plated at $5 \times 10^{4}$ cells $/ \mathrm{mL}$ per well in MSC medium and allowed to adhere for $24 \mathrm{~h}$. MSC medium was replaced with suspension medium and left for $24 \mathrm{~h}$. Subsequently, $25 \mu \mathrm{L}$ of MSC conditioned suspension medium was removed and mixed with $100 \mu \mathrm{L}$ TRIS buffer $(0.1 \mathrm{M})$ and $25 \mu \mathrm{L}$ DTNB (2.5mM, Sigma-Aldrich) solution. The reaction was left for $10 \mathrm{~min}$ at room temperature, then the absorbance read at $412 \mathrm{~nm}$. The concentration of thiols was quantified using a linear regression equation of the standard curve generated from the absorbance of known standards of cysteine.

\section{Cysteine transporter, xCT, knockdown in MSC}

Higher thiol levels are a feature of cysteine, which is usually synthesized using a specialized amino acid transport system, known as the xc system. To investigate whether knockdown of the cysteine transporter, $\mathrm{xCT}$, in MSC affected thiol production small interfering RNAs (siRNAs) for xCT and a non-coding scramble control were transfected into MSC with Lipofectamine 3000 (all Thermo Fisher Scientific) using 20nM siRNA. MSC were plated at $5 \times 10^{4}$ cells $/ \mathrm{mL}$ per well in MSC media, without Pen/Strep, containing siRNA for $24 \mathrm{~h}$. MSC media was completely removed and replaced with DMEM containing 10\% FCS for $24 \mathrm{~h}$. To assess xCT knockdown, mRNA and protein were harvested using a PARIS kit (Thermo Fisher Scientific). (See Online Supplementary Appendix for details.)

\section{Statistical analysis}

Full details of statistical analyses are provided in the Online Supplementary Appendix.

\section{Results}

\section{Cytotoxicity is linked to changes in ROS and GSH}

Eight of 10 samples responded to treatment with PTL, with a median half-maximal inhibitory concentration $\left(\mathrm{IC}_{50}\right.$ ) of $7.6 \mu \mathrm{M}$ (range 2.6-10.0 $\mu \mathrm{M}$ ). The highest dose of PTL reduced the viability of samples to an average of $30 \pm 18 \%$ compared to untreated cells (Online Supplementary Figure S2A). Two cases (patients 3 and 9) were relatively resistant to PTL with viabilities remaining above $55 \%$ at the highest dose tested (Online Supplementary Figure S2B). To measure ROS levels, cells from 4 samples were pre-incubated with the ROS sensitive probe CM H2DCFDA and then treated with PTL. After $30 \mathrm{~min}$, the level of ROS stress was significantly higher in PTL treated cells and it was 5-fold higher than untreated cells after $90 \mathrm{~min}(P \leq 0.02)$ (Figure 1A). Addition 
A

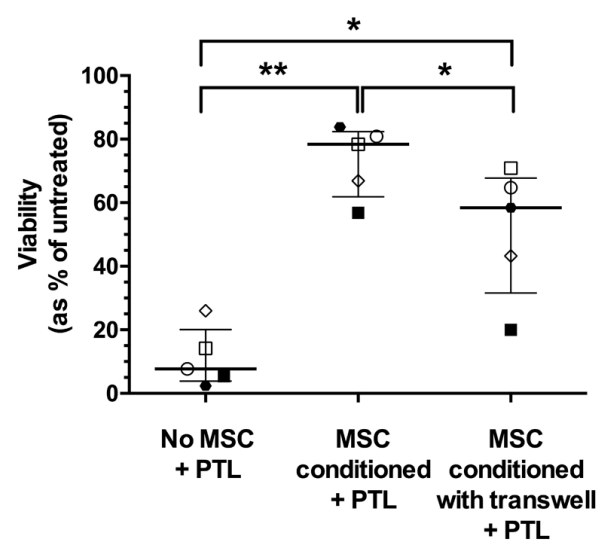

C

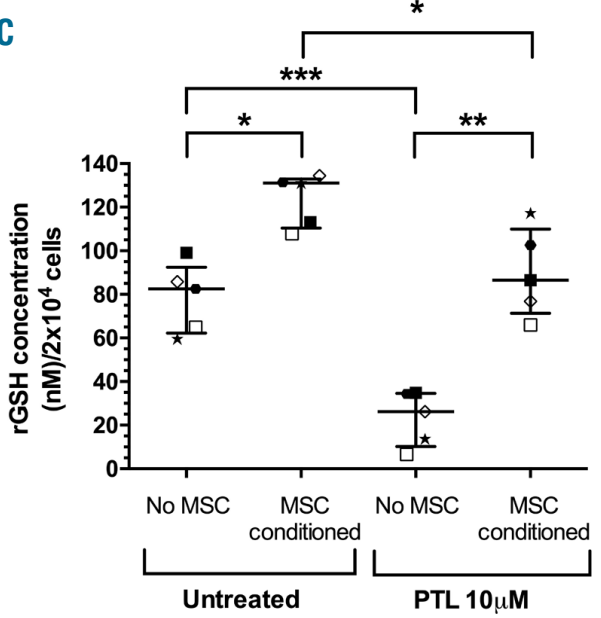

B

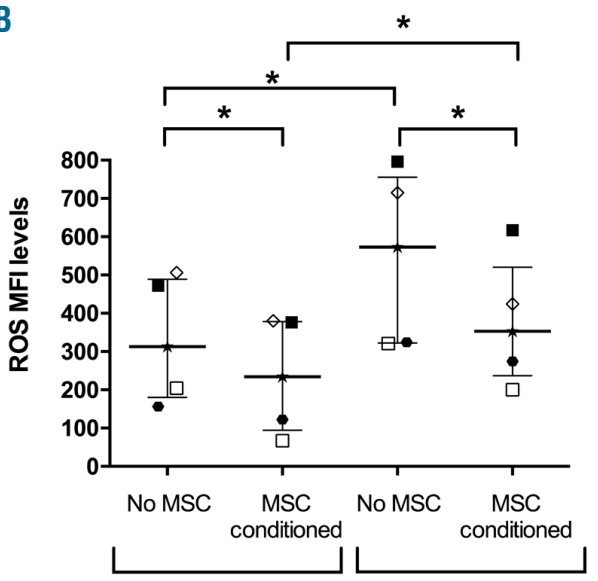

Untreated

PTL 10uM

D

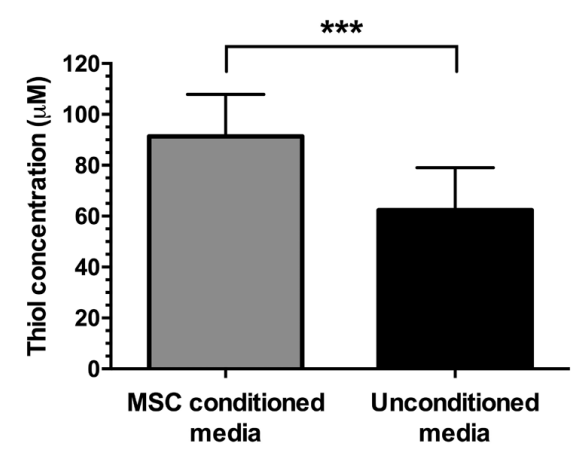

Figure 2. Mesenchymal stem cells (MSC) protect T-cell acute lymphoblastic leukemia (TALL) cells from parthenolide (PTL)-induced cell death, reactive oxygen species (ROS) stress and decreased reduced glutathione ( $\mathrm{rGSH}$ ) levels. (A) The viability of T-ALL samples (patients 1, 2, 5, 6, and 10) after 24 hours (h) of PTL treatment $(10 \mu \mathrm{M})$ with or without MSC in direct contact and in transwells. (B) ROS levels in patient samples 2, 5, 6, 9, and 10 after $1 \mathrm{~h}$ PTL treatment (10 $\mu \mathrm{M})$ with or without MSC. (C) The concentration of rGSH in patient samples 2, 5, 6, 9, and 10 after $1 \mathrm{~h}$ PTL treatment (10 $\mu \mathrm{M})$ with or without MSC. Symbols represent the average value in replicate samples. Each symbol represents an individual patient. Lines represent median and interquartile range. (D) Increase in thiol concentration in media from MSC culture after $24 \mathrm{~h}$ compared to blank media $(n=6)$. Thiol concentrations were derived from the standard curve of known cysteine levels. Results were analyzed by one-way ANOVA (AC) or paired $t$-test (D). ${ }^{*} P \leq 0.05$, $* * P \leq 0.01, * * * P \leq 0.001$. of the antioxidant NAC to PTL treated cells caused a complete block in PTL induced ROS stress over the 90-min period $(P \leq 0.02)$ (Figure $1 \mathrm{~A})$. NAC $(15 \mathrm{mM})$ reversed PTL induced cytotoxicity, even at the highest dose $(10 \mu \mathrm{M})$, where T-ALL cells retained a viability of $110 \pm 22 \%$ compared to $6 \pm 3 \%$ in cells treated with PTL alone $(P \leq 0.0001)$ (Figure 1B). A lower dose of NAC, $30 \mu \mathrm{M}$, also significantly reduced PTL cytotoxicity $(P \leq 0.01)$, increasing the $\mathrm{IC}_{50}$ from $2.3 \mu \mathrm{M}$ to $5.7 \mu \mathrm{M}$. As a further measure of oxidative changes, the levels of the anti-oxidative molecule rGSH were measured in PTL treated cells from samples 1-9. The levels of rGSH detected after $1 \mathrm{~h}$ were significantly lower in PTL treated cells with a median of $12 \%$ (range $0-58 \%$ ) compared to untreated cells $(P=0.02)$ (Figure 1C). rGSH levels were higher in the PTL resistant cases (patients 3 and 9).

\section{MSC protection from PTL and ROS stress}

Mesenchymal stem cells generated from normal BM cells were highly positive for MSC associated markers;

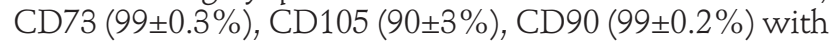
low expression of hemopoietic cell markers CD45 $(0.3 \% \pm 0.2 \%)$ and CD34 (0.6 $\pm 0.5 \%)$. MSC protected TALL cells from the cytotoxic effects of PTL (78\% median survival, range 57-84) in co-culture compared to $8 \%$ (range
$2-26 \%)$ without MSC ( $P=0.002)$ (Figure 2A). When TALL cells were seeded onto transwell inserts to prevent direct cell contact with MSC, median survival following treatment (58\%, range $20-71 \%$ ) was significantly higher compared to cells treated without MSC support $(P=0.03)$. However, T-ALL survival in transwell cultures was lower than cells in direct contact with MSC $(P=0.02)$ (Figure $2 \mathrm{~A})$. To ascertain whether this MSC conferred resistance to PTL is retained on removal from the supportive environment, T-ALL cells that had been pre-conditioned with MSC for $24 \mathrm{~h}$ were treated with PTL without further MSC support. Pre-conditioned cells showed some evidence for enhanced survival compared to cells without conditioning with an increase in $\mathrm{IC}_{50}$ from 2.6 to $3.3 \mu \mathrm{M}$. The increase in PTL resistance in MSC conditioned cells was modest but significant $(P=0.04)$ (Online Supplementary Figure S3).

As ROS levels are associated with PTL cytotoxicity, the ability of MSC to modulate ROS levels was investigated. T-ALL cells conditioned with MSC had significantly lower levels of ROS stress, (ROS MFI 234, range 67-380), compared to those without MSC conditioning (313, range 156506; $P=0.02$ ) (Figure 2B). When T-ALL cells were treated with PTL, ROS levels were significantly increased in both MSC conditioned $(P=0.04)$ and unconditioned $(P=0.01)$ cells. However, the ROS levels following PTL treatment 
A

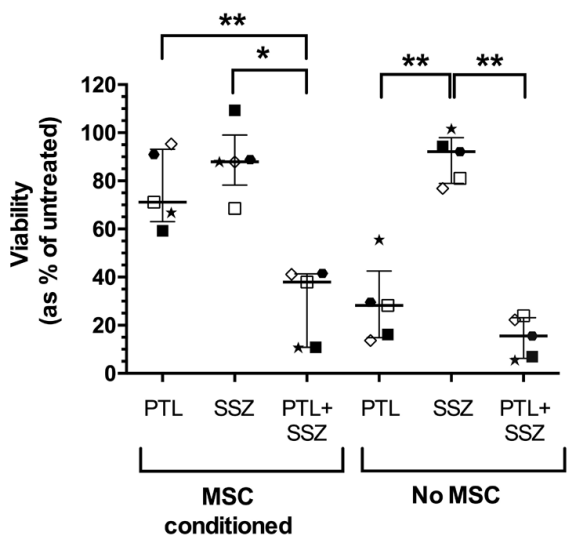

C

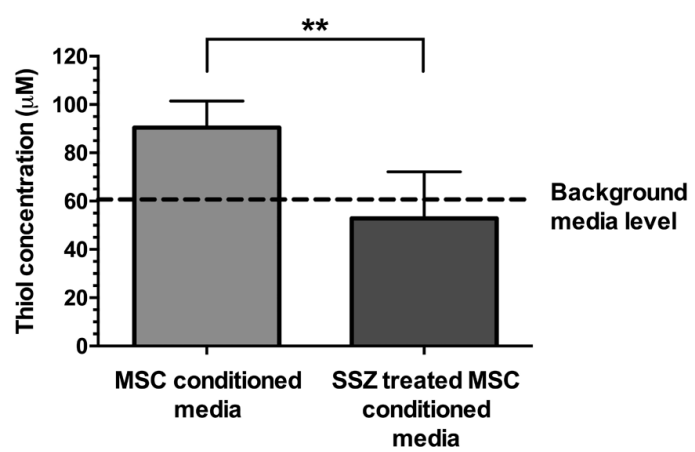

B

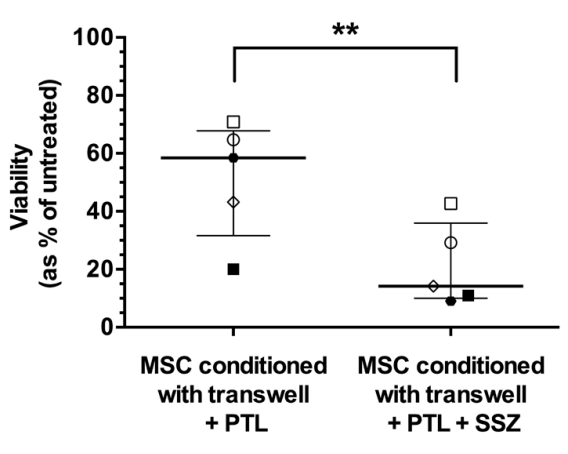

Figure 3. Combination of parthenolide (PTL) with sulfasalazine (SSZ) overcomes protective effects of mesenchymal stem cells (MSC). (A) Viability of acute lymphoblastic leukemia (ALL) samples (patients 2, 5, 6, 9, and 10) treated with PTL (10 $\mu \mathrm{M})$, SSZ $(300 \mu \mathrm{M})$ or a combination of both agents for 24 hours (h) with or without MSC. (B) Viability of samples (patients 1, 2, 5, 6, and 10) following 24-h exposure to PTL $(10 \mu \mathrm{M})$ or a combination of PTL with SSZ $(300 \mu \mathrm{M})$ separated from MSC by transwell inserts. Symbols represent the average viability in replicate samples. Each symbol represents an individual patient. Lines represent median and interquartile range. (C) Thiol concentration in media following incubation with MSC with or without SSZ treatment $(n=4)$. Data represent mean \pm Standard Deviation. Results were analyzed by one-way ANOVA (A) or paired $t$-test (B and C). ${ }^{*} P \leq 0.05, * * P \leq 0.01$. were significantly lower in MSC conditioned cells (353, range 200-617) compared to those cultured without MSC (573, range 321-796; $P=0.05$ ) (Figure $2 \mathrm{~B}$ ).

To examine whether these observed protective effects were specific to MSC, T-ALL cells were co-cultured on BM stromal cells and fibroblasts. PTL toxicity was reduced in co-cultures containing stromal cells and fibroblasts but not to the same extent as cultures containing MSC (Online Supplementary Figure S4A). T-ALL cells conditioned with stroma had similar ROS levels to those conditioned with MSC. ROS levels in cells cultured on fibroblasts were similar to those observed in unsupported cultures (Online Supplementary Figure S4B).

Parthenolide is known to lower rGSH levels in leukemia cells, therefore the ability of MSC to modulate this effect was examined. T-ALL cells conditioned with MSC had significantly higher levels of rGSH (median $131 \mathrm{nM}$, range $108-135 \mathrm{nM}$ ) compared to those without conditioning $(82 \mathrm{nM}$, range $60-99 \mathrm{nM} ; P=0.03)$. Following PTL treatment, rGSH levels were significantly decreased in both MSC conditioned $(P=0.04)$ and unconditioned $(P=0.0003)$ cells, indicating reduced anti-oxidant activity. However, rGSH levels were still higher in MSC conditioned cells (median $87 \mathrm{nM}$, range $66-117 \mathrm{nM}$ ) compared to those without MSC conditioning (26nM, range 7-35nM; $P=0.01$ ) (Figure 2C).

As cysteine is crucial for GSH synthesis in leukemia, it is possible that MSC release cysteine into the surrounding media, thereby moderating the anti-oxidant effects of PTL. A thiol detection assay was used, since cysteine contains a free thiol group, to investigate cysteine release. Thiol concentrations were significantly higher in media removed from MSC cultures $(91 \pm 17 \mu \mathrm{M})$ compared to background media levels $(62 \pm 17 \mu \mathrm{M} ; P=0.0002)$, representing a 1.5-fold increase over background (Figure 2D).

\section{Blocking thiol release overcomes protective effect}

The thiol functional group plays a major role in intracellular anti-oxidant defenses. Cysteine residues eliminate ROS, usually by converting them to $2 \mathrm{H}_{2} \mathrm{O}$ or $\mathrm{H}_{2} \mathrm{O}$ and $\mathrm{O}_{2}$, and reduce oxidized protein thiols. ${ }^{14} \mathrm{SSZ}$, an inhibitor of the cystine uptake antiporter xc', was used to inhibit cystine uptake and block subsequent cysteine generation and release in MSC, to determine what effect this would have on the protection conferred by MSC. The combination of PTL and SSZ significantly reduced the median viability of T-ALL cells with MSC conditioning to 38\% (range 11 $42 \%$ ) compared to PTL (71\%, range $59-95 \%$; $P=0.002)$ or SSZ alone (88\%, range $69-109 \% ; P=0.04)$ (Figure 3A). The treatment combination reduced viabilities to levels comparable to those observed in cells treated with both agents without MSC conditioning (16\%, range 5-24\%). In the absence of MSC, SSZ had low cytotoxicity ( $92 \%$ median survival, range $77-102 \%$ ) and when combined with PTL there was no significant difference in toxicity compared to PTL alone $(P=0.9)$. Addition of PTL+ SSZ to T-ALL cells conditioned with BM stroma or fibroblasts also reduced leukemia cell survival, although to a lesser extent (Online Supplementary Figure S4C). Interestingly, the effects of combining these agents were more remarkable in samples that were relatively resistant to PTL. Fiftysix percent of cells from patient 9 survived treatment with PTL, but viability was reduced to only $6 \%$ when PTL was combined with SSZ. Leukemia burden in NSG mice engrafted with this sample was significantly reduced when treated with PTL and SSZ compared to placebos 
A

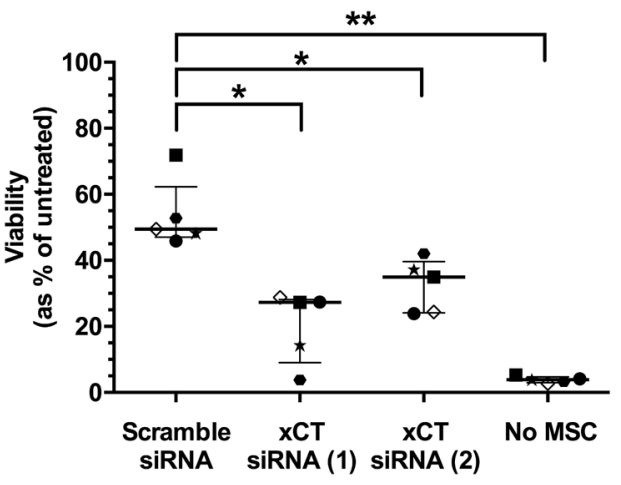

C

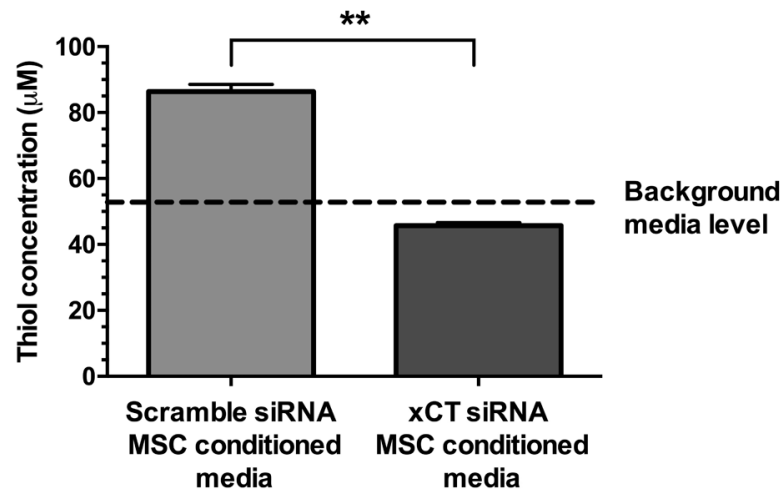

B

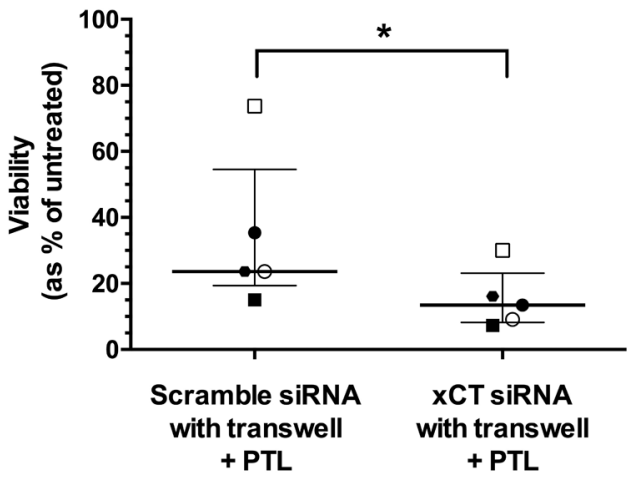

Figure 4. xCT knockdown in mesenchymal stem cells (MSC) overcomes MSC mediated resistance to parthenolide (PTL). Viability of T-cell acute lymphoblastic leukemia (T-ALL) samples (patients 5, 6, 8-10) treated with $10 \mu \mathrm{M}$ PTL for 24 hours (h) in the presence of MSC pre-treated with xCT or scramble control siRNA, in direct contact (A) or samples $(1,2,6,8$, and 10 ) in transwell inserts (B). Symbols represent the average viability in duplicate samples. Each symbol represents an individual patient. Lines represent median and interquartile range. (C) Thiol concentration in media following 24-h incubation with MSC pre-treated with xCT or scramble control siRNA compared to background media. Data represent mean \pm Standard Deviation. $(n=4)$. Results were analyzed by one-way ANOVA (A) or paired $t$-tests (B and C). ${ }^{*} P \leq 0.05, * * P \leq 0.01$.
$(P<0.0001)$ (Online Supplementary Figure S5). Using SSZ alone and in combination with PTL had minimal effects on normal hemopoietic cells (Online Supplementary Figure S6).

In transwell experiments, using SSZ with PTL abrogated the protective effects of MSC support, reducing T-ALL survival from $58 \%$ (range $20-71 \%$ ) to $14 \%$ (range $9-43 \%$; $P=0.01$ ) (Figure 3B). Media harvested from cultures of SSZ treated MSC contained thiol levels similar to background media $(53 \pm 19 \mu \mathrm{M} v s .61 \pm 10 \mu \mathrm{M}$, respectively). In contrast, media from untreated MSC contained significantly higher thiol levels $(90 \pm 11 \mu \mathrm{M} ; P=0.005)$ compared to media from SSZ treated cells, representing a 1.5-fold increase over background media (Figure 3C).

As a second approach to block the cystine uptake antiporter $\mathrm{xc}^{\prime}$, and thereby prevent cysteine release from MSC, xCT was targeted with siRNAs. Relative gene expression levels of $\mathrm{xCT}$ were significantly lower in MSC $48 \mathrm{~h}$ after transfection with either siRNA $1(17 \pm 9 \%)$ or siRNA $2(9 \pm 4 \%)$ compared to scramble control siRNA $(P \leq 0.0001)$ (Online Supplementary Figure S7A). Protein available from $\mathrm{xCT}$ siRNA-1 showed a significant reduction in $\mathrm{xCT}$ expression $(3 \pm 0.1 \%)$ relative to scramble control levels quantified by western blotting densitometry $(P=0.002)$ (Online Supplementary Figure S7B).

The viability of PTL treated T-ALL cells, co-cultured with MSC treated with xCT siRNA-1 (27\%, range 3-28\%) or $-2(35 \%$, range $24-42 \%)$, was significantly lower than cells co-cultured with scramble control MSC (49\%, range $46-72 \% ; P<0.05$ ) (Figure 4A). As siRNA-1 provided the best reduction in MSC protection, it was selected for further experiments. When transwell inserts were used to separate xCT knockdown MSC from T-ALL cells, the median viability after PTL treatment was significantly lower $(13 \%$, range $7-30 \%)$ compared to scramble control MSC cells ( $24 \%$, range $15-74 \%$; $P=0.05$ ) (Figure 4B). Scramble control MSC media had a significantly higher thiol concentration $(86 \pm 2 \mu \mathrm{M})$ compared to media harvested from xCT knockdown MSC $(46 \pm 1 \mu \mathrm{M} ; P=0.002)$ (Figure 4C). Media from xCT knockdown MSC did not show any increase in thiol levels above background media $(53 \pm 1 \mu \mathrm{M})$.

To confirm that the observed effects of interfering with xCT were a result of blocking cystine uptake, and not a result of toxicity, the viability and morphology of treated MSC were assessed. Treatment with PTL, SSZ or both agents in combination had no effect on the viability and confluency of MSC $(P \geq 0.37)$ (Figure $5 \mathrm{~A})$ nor did siRNA treatment $(P \geq 0.82)$ (Figure $5 \mathrm{~B}$ and Online Supplementary Figure S8). This was also confirmed by flow cytometry (Figure 5C).

\section{Discussion}

To date, PTL is the only drug that has been shown to be capable of completely eradicating childhood ALL in NSG xenografts, as a single agent. ${ }^{8}$ Most studies, using such models, report reduction in leukemia burden but levels often increase on cessation of treatment. Consequently, there is much interest in the application of PTL for cancer therapy. Several groups are developing strategies to improve the bioavailability of PTL, without having detrimental effects on its pharmacokinetic properties. ${ }^{15-19}$ PTL can be successfully sequestered into nanoscopic vectors which can achieve equivalent toxicity to unmodified PTL. ${ }^{16}$ ${ }^{18}$ Nanoparticle PTL formulations can be used in vivo at 40fold lower doses with 20-fold lower administration fre- 
A

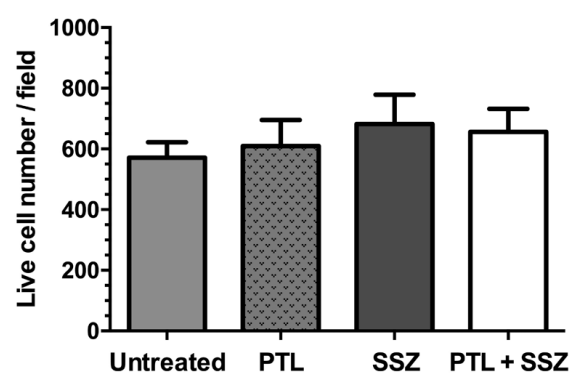

B

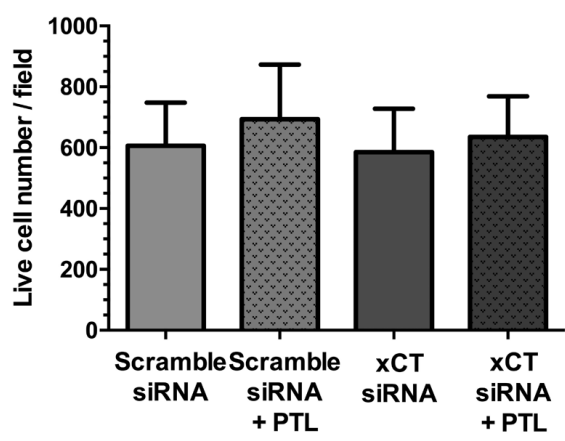

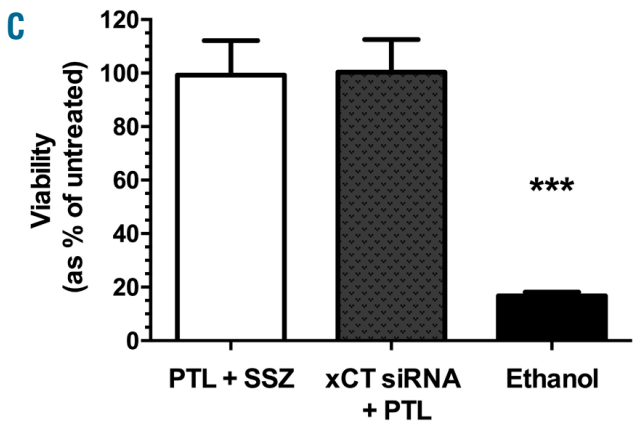

Figure 5. Effects of parthenolide (PTL) and xCT modifying agents on mesenchymal stem cell (MSC) viability. (A) Average live MSC counts from 3 fields (x10 magnification) following treatment with PTL $(10 \mu \mathrm{M})$, sulfasalazine (SSZ) $(300 \mu \mathrm{M})$, or a combination of both agents measured by fluorescence microscopy. (B) Average live MSC counts from 3 fields (x10 magnification) following treatment with PTL $(10 \mu \mathrm{M})$, pre-treated with $\mathrm{xCT}$, or scramble control siRNA. (C) Viability of MSC following treatment with PTL $(10 \mu \mathrm{M})$ and SSZ $(300 \mu \mathrm{M})$, or PTL after pre-treatment with xCT siRNA, measured by flow cytometry. Treatment with $70 \%$ ethanol served as a positive control for inducing toxicity. Data represent mean \pm Standard Deviation $(n=3)$. Live cell counts by fluorescence microscopy were calculated using the ImageJ particle analysis software. Results were analyzed by one-way ANOVA (A-C). $* * * P \leq 0.001$.

quency than standard PTL. ${ }^{16}$ Some of these delivery systems are inexpensive and formulations are readily scalable. ${ }^{18}$ Consequently, they should facilitate the use of PTL at clinically relevant doses. However, the efficacy of many therapeutic drugs may be compromised by the host BM microenvironment. Therapy-induced niches, which protect leukemia cells against standard first-line induction agents, have been described in ALL..$^{12,20}$ In the present study, we investigated whether resistance to PTL, reported in a minority of T-ALL cases, ${ }^{8}$ may be conferred by BM-derived MSC.

In vitro PTL treatment reduced viability of T-ALL cells to less than $30 \%$, confirming previous results in a separate cohort of pediatric cases. ${ }^{8}$ While there were differences in the responses of individual patient samples, there was no correlation between PTL cytotoxicity with karyotype or $\mathrm{MRD}$ risk status, which may be a result of the heterogeneous nature of this disease. PTL was shown to increase ROS stress and lower rGSH levels in T-ALL. The antioxidative compound NAC blocked ROS upregulation and diminished PTL cytotoxicity, suggesting that PTL toxicity is, at least in part, related to ROS stress in T-ALL. These findings concur with reports of increased ROS stress in primary AML and CLL cell lines following PTL treatment. ${ }^{6,1,22}$

The protective effect provided by NAC may be attributed to the role of cysteine as the rate limiting amino acid in rGSH production. ${ }^{23}$ Reduction of cystine to cysteine and subsequent supply to leukemia cells is crucial for GSH synthesis in these cells. GSH levels elevate the anti-oxidative capacity of cells, which may provide protection against PTL. In AML and breast cancer, populations of cells endure lower levels of ROS stress and these cells are more resistant to therapy. ${ }^{24,25}$ It is unclear whether ROS is actively eliminated by rGSH or if PTL directly interacts with rGSH, leading to a reduction in anti-oxidative capacity and increased ROS accumulation. One function of rGSH is to detoxify cells from reactive electrophiles ${ }^{26}$ which PTL contains in the form of an $\alpha, \beta$-unsaturated carbonyl group. PTL can directly interfere with rGSH synthesis in AML, causing depletion of rGSH, allowing ROS levels to increase. ${ }^{21}$ Whether PTL directly blocked rGSH synthesis was beyond the scope of this study. However, it is evident that ROS levels increased and rGSH levels dropped, putting the cells under higher levels of stress, which is likely to drive apoptosis.

As these data indicate that ROS levels may be linked to PTL cytotoxicity, it is possible that the BM microenvironment provides resistance to drug activity by protecting leukemia cells from ROS stress. CLL cells had decreased sensitivity to the ROS inducing agents fludarabine and oxiplatin when co-cultured with BM stromal cells. ${ }^{9}$ Chemoprotection was derived from the generation and release of cysteine by stromal cells for uptake by CLL cells. The BM microenvironment can also play a role in leukemia drug resistance, mediated by a diverse set of mechanisms, both by direct cell adhesion ${ }^{11,13,27}$ and/or soluble factor release. ${ }^{12,20,28,29}$ In this study, the protective effects of MSC against PTL toxicity were related to decreases in ROS stress and preserved rGSH levels, suggesting that MSC increased the anti-oxidative stress capacity of T-ALL cells and thereby conferred resistance to PTL. To eliminate variability, MSC were generated from a single normal BM donor, allowing direct comparison of the effects on the patients' samples. This may have introduced bias but our results are consistent with those reported in CLL. ${ }^{9}$ These data also suggest that protection is provided by one or more secretable factors, as preventing direct contact with MSC still provided $\mathrm{T}$ ALL cells with protection against PTL. However, protection was not to the same extent as observed in direct contact, suggesting involvement of other factors.

Mesenchymal stem cell conditioned media contained significantly higher levels of thiols, an important feature of the anti-oxidative compound cysteine, compared to unconditioned media. A key mechanism by which normal cells synthesize cysteine is via intake of extracellular cystine 


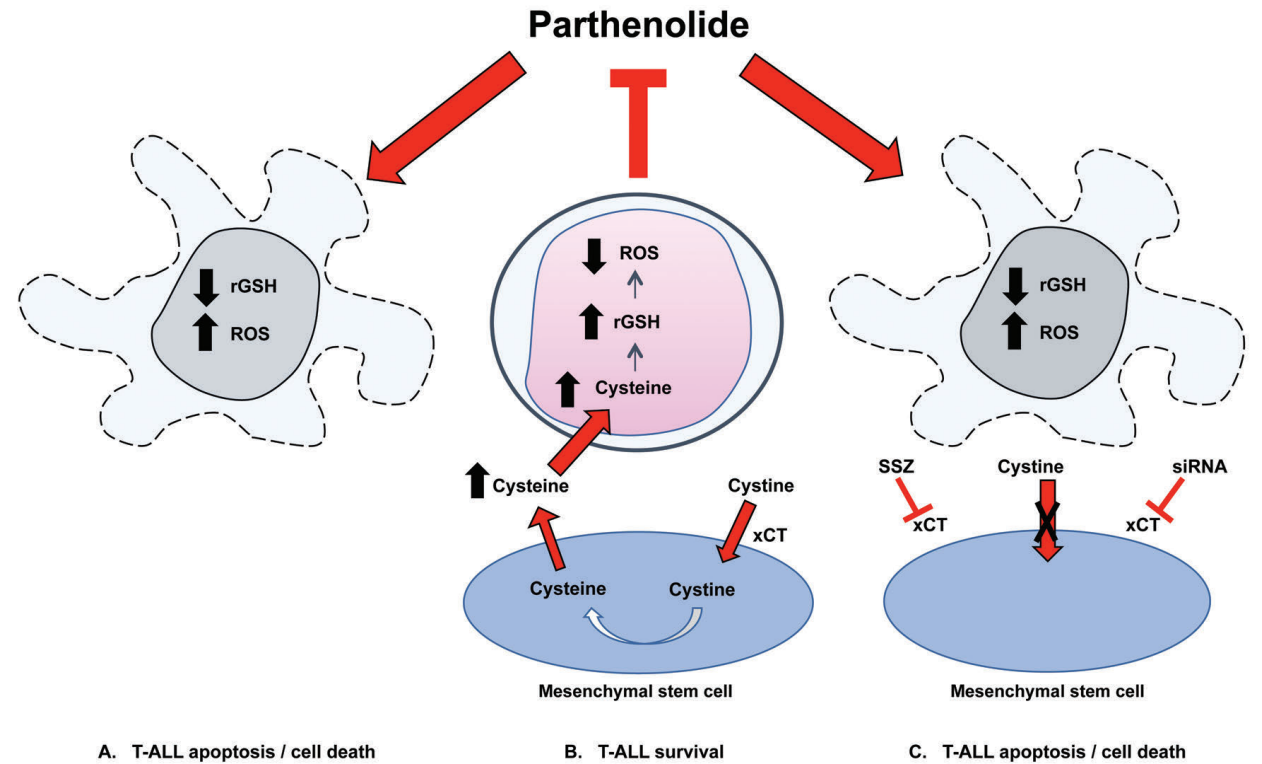

Figure 6. Proposed mechanism of action of parthenolide (PTL) and the protective effect provided by mesenchymal stem cells (MSC). PTL causes apoptosis by increasing reactive oxygen species (ROS) stress and decreasing reduced glutathione $(\mathrm{rGSH})$ resulting in death of T-cell acute lymphoblastic leukemia (T-ALL) cells (A). MSC express high levels of the cystine glutamate antiporter xc-, facilitated by the antiporter protein XCT. Extracellular cystine is taken up by MSC and reduced into cysteine. Cysteine is released into the extracellular space for uptake by T-ALL cells, blocking ROS induction and preventing apoptosis (B). Blocking the $\mathrm{xCT}$ system using sulfasalazine (SSZ) or siRNA reduces protective effects provided by MSC (C).

using a specialized amino acid transport mechanism known as the $\mathrm{xc}^{-}$system. ${ }^{14}$ The system specifically mediates cystine and glutamate exchange across the cell membrane and is comprised of two subunits: the light chain transporter subunit SLC7A11 (or xCT) and the heavy subunit SLC3A2 (4F2hc). ${ }^{30,31}$ Cysteine is unstable outside of cells and is rapidly oxidized to form a disulphide bridge with another cysteine amino acid, forming cystine. However, upon entry into the cell, cystine enters a stronger reducing environment and is converted into cysteine. Blocking Xc activity with a small molecule inhibitor, $\mathrm{SSZ},{ }^{32}$ or by knockdown of the $\mathrm{xc}^{-}$ light chain component $\mathrm{xCT}$, prevented thiol release and significantly reduced MSC protection to levels close to those observed in cells without MSC support. In addition, leukemia cell survival in a PTL resistant case was significantly decreased when SSZ or XCT siRNA were used to block xc- activity. Furthermore, PTL and SSZ treatment in vivo resulted in significantly reduced leukemia burden in engrafted NSG mice compared to controls. Similar results have been reported in BCP-ALL, where cell viability was diminished in a subset of patients following treatment with cysteine dioxygenase, which catalyzes conversion of cysteine into cysteine sulfinic acid, thereby bypassing GSH synthesis. ${ }^{33}$ Together, these findings indicate that MSC release cysteine, which confers a survival advantage on leukemia cells. The functional contribution of thiols was further confirmed by the demonstration that T-ALL cells exposed to the thiol containing compound NAC, at an equivalent thiol concentration to that in MSC conditioned media, were more resistant to PTL. The fact that there was still a small protective effect against PTL after interfering with the xc system, indicates that cysteine release may not be the sole protective mechanism in these experiments. A recent study found that ALL cells release extracellular vesicles that can be taken up by MSC, causing a shift to glycolysis metabolism. The switch to glycolysis led to an increased release of the metabolite lactate, which might be used as an additional source of energy by leukemia cells and confer chemoresistance..$^{34}$

While MSC are a fundamental component of the BM niche, and contribute to its formation in vivo, other stromal cells may have a role in leukemia maintenance and protection from therapeutic agents. We demonstrated that BM stromal cells and fibroblasts also conferred protection against PTL. ALL cells themselves can alter the endosteal and vascular compartments of the niche ${ }^{12}$ and induce apoptosis of osteoblast cells. ${ }^{17}$ The remodelled niche is dynamically transient and on exposure to chemotherapeutic agents, ALL cells can release CCL3 and cytokines TGF- $\beta 1$ and GDF15, conferring chemoresistance by activating the TGF- $\beta$ signaling pathway. ${ }^{12}$ It may be possible to overcome this resistance by disrupting the interactions between leukemia cells and the BM environment.

This is the first report demonstrating MSC provide a protective effect to T-ALL cells against PTL. Moreover, we have shown that targeting the xc' system can overcome this effect (see overview in Figure 6), adding to the evidence that targeting $\mathrm{xc}^{-}$enhances the efficacy of anti-cancer agents in leukemias ${ }^{9}$ and in several solid cancer models. ${ }^{35.38}$ MSC viability was unaffected by PTL and targeting $\mathrm{xc}$, so damage to this important element of the BM environment $^{39,40}$ should be minimal. A logical progression of this work will be determining whether the effects of PTL, or indeed current chemotherapeutic agents, in vivo can be enhanced using an xc inhibitor like SSZ. Furthermore, with developments in nanoscopic drug delivery vectors, it may be possible to use dual loaded (PTL+SSZ) nanovectors to overcome selective patient resistance towards one drug and the chemoprotective effects of the leukemia microenvironment in vivo.

\section{Acknowledgments}

The authors would like to thank Dr Jeremy Hancock, Mr Paul Virgo and staff of Bristol Genetics Laboratory, Southmead Hospital for excellent technical assistance. We also thank Elinor Curnow, Statistics and Clinical Studies, NHS Blood and Transplant, consultants and oncology staff at Bristol Royal Hospital for Children. We are grateful to the patients and their families who gave permission for their cells to be used for research. This work was supported by a generous donation from Mr Richard Cunningham and by grants from the Department of Health and NHS Blood and Transplant. 


\section{References}

1. Schrappe $\mathrm{M}$, Hunger SP, Pui $\mathrm{CH}$, et al Outcomes after induction failure in childhood acute lymphoblastic leukemia. N Engl J Med. 2012;366(15):1371-1381.

2. Hunger SP, Mullighan CG. Acute Lymphoblastic Leukemia in Children. N Engl J Med. 2015;373(16):1541-1552.

3. Trachootham D, Alexandre J, Huang P. Targeting cancer cells by ROS-mediated mechanisms: a radical therapeutic approach? Nature Rev Drug Discov. 2009;8(7):579-591.

4. Silva A, Yunes JA, Cardoso BA, et al. PTEN posttranslational inactivation and hyperactivation of the PI3K/Akt pathway sustain primary $\mathrm{T}$ cell leukemia viability. J Clin Invest. 2008;118(11):3762-3774.

5. Giambra V, Jenkins CR, Wang $H$, et al. NOTCH1 promotes $\mathrm{T}$ cell leukemia-initiating activity by RUNX-mediated regulation of PKC-theta and reactive oxygen species. Nat Med. 2012;18(11):1693-1698.

6. Guzman ML, Rossi R, Karnischky L, et al. The sesquiterpene lactone parthenolide induces apoptosis of human acute myelogenous leukemia stem and progenitor cells. Blood. 2005;105(11):4163-4169.

7. Steele AJ, Jones DT, Ganeshaguru K, et al. The sesquiterpene lactone parthenolide induces selective apoptosis of B-chronic lymphocytic leukemia cells in vitro. Leukemia. 2006:20(6):1073-1079.

8. Diamanti P, Cox CV, Moppett JP, Blair A. Parthenolide eliminates leukemia-initiating cell populations and improves survival in xenografts of childhood acute lymphoblastic leukemia. Blood. 2013;121(8):1384-1393.

9. Zhang W, Trachootham D, Liu J, et al Stromal control of cystine metabolism promotes cancer cell survival in chronic lymphocytic leukaemia. Nat Cell Biol. 2012;14(3):276-286.

10. Wu KN, Zhao YM, He Y, et al. Rapamycin interacts synergistically with idarubicin to induce T-leukemia cell apoptosis in vitro and in a mesenchymal stem cell simulated drugresistant microenvironment via Akt $/ \mathrm{mam}$ malian target of rapamycin and extracellular signal-related kinase signaling pathways. Leuk Lymphoma. 2014;55(3): 668-676.

11. Jacamo R, Chen Y, Wang Z, et al. Reciprocal leukemia-stroma VCAM-1/VLA-4-dependent activation of NF-kappaB mediates chemoresistance. Blood. 2014;123(17):26912702.

12. Duan CW, Shi J, Chen J, et al. Leukemia propagating cells rebuild an evolving niche in response to therapy. Cancer Cell. 2014:25(6):778-793.

13. Xia B, Tian C, Guo S, et al. c-Myc plays part in drug resistance mediated by bone marrow stromal cells in acute myeloid leukemia. Leuk Res. 2015;39(1):92-99.

14. Lewerenz J, Hewett SJ, Huang Y, et al. The cystine/glutamate antiporter system $\mathrm{x}(\mathrm{c})(-)$ in health and disease: from molecular mechanisms to novel therapeutic opportunities. Antioxid Redox Signal. 2013;18(5):522-555.

15. Baranello MP, Bauer L, Jordan CT, Benoit DSW. Micelle Delivery of Parthenolide to Acute Myeloid Leukemia Cells. Cell Mol Bioneng. 2015;8(3):455-470.

16. Zong H, Sen S, Zhang G, et al. In vivo targeting of leukemia stem cells by directing parthenolide-loaded nanoparticles to the bone marrow niche. Leukemia. 2016;30(7): 1582-1586.

17. Deller RC, Diamanti P, Morrison G, et al. Functionalized Triblock Copolymer Vectors for the Treatment of Acute Lymphoblastic Leukemia. Mol Pharm. 2017;14(3):722-732.

18. Ridolfo R, Ede B, Diamanti P, et al. Drug loaded nanovectors via direct hydration as a new platform for cancer therapeutics. Small. 2018 Jul 12:e1703774. doi: 10.1002/ smll.201703774. [Epub ahead of print]

19. Tyagi V, Alwaseem H, O'Dwyer KM, et al. Chemoenzymatic synthesis and antileukemic activity of novel C9- and C14functionalized parthenolide analogs. Bioorg Med Chem. 2016;24(17):3876-3886.

20. Hawkins ED, Duarte D, Akinduro O, et al. T-cell acute leukaemia exhibits dynamic interactions with bone marrow microenvironments. Nature. 2016;538(7626):518-522.

21. Pei S, Minhajuddin M, Callahan KP, et al. Targeting aberrant glutathione metabolism to eradicate human acute myelogenous leukemia cells. J Biol Chem. 2013;288 (47):33542-33558.

22. Zunino SI, Ducore JM Storms DH. Parthenolide induces significant apoptosis and production of reactive oxygen species in high-risk pre-B leukemia cells. Cancer Lett. 2007;254(1):119-127.

23. Lu SC. Glutathione synthesis. Biochim Biophys Acta. 2013;1830(5):3143-3153.

24. Lagadinou ED, Sach A, Callahan K, et al. BCL-2 inhibition targets oxidative phosphorylation and selectively eradicates quiescent human leukemia stem cells. Cell Stem Cell. 2013;12(3):329-341.

25. Diehn M, Cho RW, Lobo NA, et al. Association of reactive oxygen species levels and radioresistance in cancer stem cells. Nature. 2009;458(7239):780-783

26. Hayes JD, Flanagan JU, Jowsey IR. Glutathione transferases. Annu Rev Pharmacol Toxicol. 2005:45:51-88.

27. Winter SS, Sweatman JJ, Lawrence MB, Rhoades TH, Hart AL, Larson RS. Enhanced T-lineage acute lymphoblastic leukaemia cell survival on bone marrow stroma requires involvement of LFA- 1 and ICAM-1. Br J Haematol. 2001;115(4):862-871.

28. Iwamoto S, Mihara K, Downing JR, Pui CH, Campana D. Mesenchymal cells regulate the response of acute lymphoblastic leukemia cells to asparaginase. J Clin Invest.
2007;117(4):1049-1057

29. Laranieira AB, de Vasconcellos JF, Sodek L, et al. IGFBP7 participates in the reciprocal interaction between acute lymphoblastic leukemia and BM stromal cells and in leukemia resistance to asparaginase. Leukemia. 2012;26(5):1001-1011.

30. Sato H, Tamba M, Ishii T, Bannai S. Cloning and expression of a plasma membrane cystine/glutamate exchange transporter composed of two distinct proteins. J Biol Chem 1999;274(17):11455-11458.

31. Sato H, Tamba M, Kuriyama-Matsumura K, Okuno S, Bannai S. Molecular cloning and expression of human $\mathrm{xCT}$, the light chain of amino acid transport system xc. Antioxid Redox Signal. 2000;2(4):665-671.

32. Aoki Y, Watanabe T, Saito Y, et al Identification of CD34+ and CD34 leukemia-initiating cells in MLL-rearranged human acute lymphoblastic leukemia. Blood. 2015;125(6):967-980.

33. Boutter I, Huang Y, Marovca B, et al. Imagebased RNA interference screening reveals an individual dependence of acute lymphoblastic leukemia on stromal cysteine support. Oncotarget. 2014;5(22):11501-11512.

34. Johnson SM, Dempsey C, Chadwick A, et al. Metabolic reprogramming of bone marrow stromal cells by leukemic extracellular vesicles in acute lymphoblastic leukemia. Blood. 2016;128(3):453-456

35. Yoshikawa M, Tsuchihashi $\mathrm{K}$, Ishimoto $\mathrm{T}$, et al. xCT inhibition depletes CD44v-expressing tumor cells that are resistant to EGFRtargeted therapy in head and neck squamous cell carcinoma. Cancer Res. 2013;73(6):1855-1866

36. Balza E, Castellani P, Delfino L, Truini M, Rubartelli A. The pharmacologic inhibition of the $\mathrm{xc}^{-}$antioxidant system improves the antitumor efficacy of COX inhibitors in the in vivo model of 3-MCA tumorigenesis. Carcinogenesis. 2013;34(3):620-626.

37. Lanzardo S, Conti L, Rooke R, et al Immunotargeting of Antigen $\mathrm{xCT}$ Attenuates Stem-like Cell Behavior and Metastatic Progression in Breast Cancer. Cancer Res. 2016;76(1):62-72.

38. Liu R, Blower PE, Pham AN, et al. Cystineglutamate transporter SLC7A11 mediates resistance to geldanamycin but not to 17 (allylamino)-17-demethoxygeldanamycin Mol Pharmacol. 2007;72(6):1637-1646.

39. Le Blanc K, Samuelsson H, Gustafsson B, et al. Transplantation of mesenchymal stem cells to enhance engraftment of hematopoietic stem cells. Leukemia. 2007;21(8):1733 1738.

40. Koc ON, Gerson SL, Cooper BW, et al. Rapid hematopoietic recovery after coinfusion of autologous-blood stem cells and culture-expanded marrow mesenchymal stem cells in advanced breast cancer patients receiving high-dose chemotherapy. I Clin Oncol. 2000;18(2):307-316 